\title{
Ideal Observer Model for Detection of Blood Perfusion and Flow Using Ultrasound
}

\author{
Roger J. Zemp ${ }^{1}$, Craig K. Abbey, Michael F. Insana \\ Department of Biomedical Engineering, University of California, Davis \\ Davis, CA 95616
}

\begin{abstract}
An ideal observer model is developed for the task of detecting blood perfusing or flowing through tissue. The ideal observer theory relies on a linear systems model that describes tissue and blood object functions and electronic noise as random processes. When aliasing is minimal, the system is characterized by a quantity similar to Noise-Equivalent Quanta used in photon imaging modalities. A simple 1-D model is used to illustrate the effect of the system and object parameters on task performance. Velocity and decorrelation are seen to be advantageous for detection. Aliasing can degrade performance. The ideal observer model provides a framework for assessing the performance of Power Doppler ultrasound systems, and may aid in their design.
\end{abstract}

\section{Introduction}

Estimation of blood velocity has been widely dealt with in the medical ultrasound literature [1]. Less has been done on detection of blood. Our motivation for studying detection is angiogenesis in tumors. Increased microvascular density and metabolic demand have been correlated with malignant growth. In many cases this means a greater flow rate through capillary networks. Various techniques have been used to quantify blood volume fraction and flow rates to monitor tumor progression [2]. Perfusion quantification could be an extension of this work, but we focus early detection of neoplasm perfusion with ultrasonic techniques.

Color Doppler Ultrasound systems display mean velocity estimates of blood motion, and have been effective for looking at larger vessels. These systems typically send a sequence of pulses and measure inter-pulse motion to estimate flow. The systems consist of a wall filter to reject stationary tissue clutter, and a mean velocity estimator that often relies on lag- 0 and lag-1 autocorrelation estimates [3]. Cross-correlation techniques also exist, some of which provide sensitivity to velocity in all directions [4]. Unfortunately, color Doppler systems are not very effective for studying perfusion in the microvasculature where in a single sample volume blood may be moving in multiple

\footnotetext{
${ }^{1}$ Electronic Mail: rjzemp@ucdavis.edu
} 
directions, giving a zero mean velocity estimate. Slow flow in perfusion-based models is another problem since clutter and blood spectra will often overlap.

Power Doppler systems [5] do not attempt to estimate velocity but rather display the signal power from blood that is moving. Hence, Power Doppler systems are often much more effective for detection of blood than Color Doppler techniques. Power Doppler is approximately independent of blood-flow velocity and Doppler angle, provided the Doppler frequency shift is non-zero. Another important feature of Power Doppler estimates for microvascular flow imaging is that they are not subject to aliasing, enabling use of a lower pulse repetition frequency $(P R F)$ than that necessary in color Doppler. Power Doppler ultrasonography is therefore more sensitive to slow flow than color Doppler. Spectral integration means that noise is also less of a problem than in color Doppler. High gain settings are therefore possible with power Doppler. Tissue motion is however a problem. Tissue clutter will tend to bias the result, dominating the total energy. Wall filters may be used to partially reject tissue clutter. Power Doppler systems can use lag-0 autocorrelation estimates already computed for Color Doppler mode imaging. The post-wall filtered lag-0 autocorrelation simply gives the power in the moving signal, by Parseval's theorem.

Despite wide commercial implementation, very little has been written in the literature about engineering approaches used in Power Doppler, and there are no first principle performance assessment techniques for Power Doppler systems. This is our contribution. We provide a framework for modeling the ideal observer for detection of blood. The ideal observer provides the upper bound on detection performance for a Power Doppler system. It can be applied to standard measurement approaches to evaluate system design and wall filters, and it provides a framework for improvements.

\section{Modeling Perfusion and Echo Signals}

\subsection{Signal Models}

In Doppler ultrasound systems ensembles of $L$ pulses are sent in sequence along axial lines of sight at a rate called the pulse repetition frequency $(P R F)$. The echoes from each pulse return to the transducer following their propagation and scattering by microstructures in the body. The measured voltage trace from a single pulse is indicative of scattering strength as a function of depth. The voltage signal is sometimes called radiofrequency (RF) data because it has an underlying modulation (due to the transmitted pulse) in the frequency range of MHz. The modulation of the signal allows for phase sensitive measurements to be made. Motion between pulses can be used to estimate velocity. With $L$ pulses, there will be $L$ voltage traces initiated at times $t_{1}, \ldots, t_{\mathrm{L}}$, where $t_{\mathrm{k}}$ $=(k-1) \Delta t, k=1, \ldots, L$, and $\Delta t$ is the pulse repetition interval $(P R I)$. These are called slow times. Voltage traces for each pulse are acquired in fast time. Fast time acquisition rates $f_{s}$ 
are usually 10 times the modulation frequency or better, whereas slow time pulserepetition frequencies are more typically in the order of magnitude of $1-100 \mathrm{KHz}$. A voltage trace for each pulse sent is collected before the next pulse is sent. After the entire ensemble of pulses is sent along one line of sight, the beam is translated mechanically or 'steered' electronically (using phased array transducers) to interrogate an adjacent line of sight with another ensemble of pulses (Fig. 1).

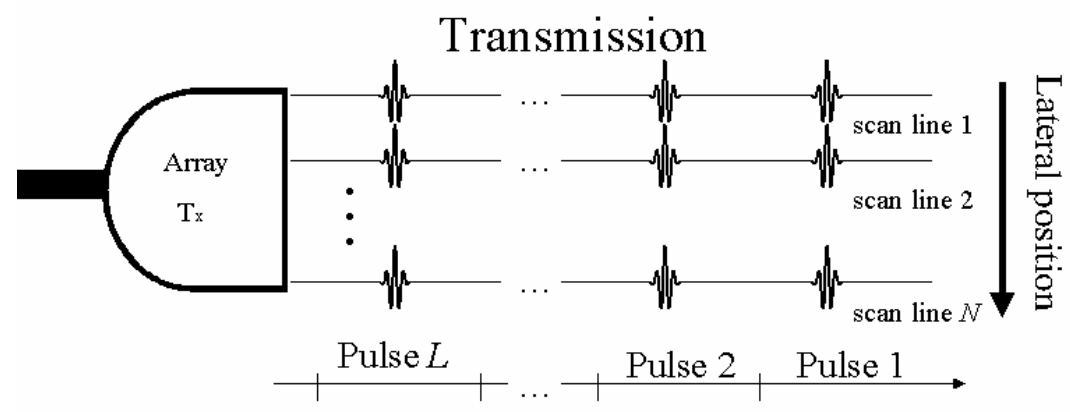

Fig. 1. Illustration of pulse sequence of a typical Doppler ultrasound system

To investigate estimation and detection problems, we assume that echo signals due to Doppler pulse sequences may be modeled as a shift-invariant linear system. While ultrasound systems have spatially varying beam properties, many modern phased array systems use special techniques such as dynamic focusing to obtain a reasonably uniform resolution cell throughout much of the image. We assume that the ultrasound system can take a sequence of 'snapshot images' of the object, and that the object can vary over time.

$r\left(\mathbf{x}, t_{k}\right)=h(\mathbf{x}) *\left[z_{b}\left(\mathbf{x}, t_{k}\right)+z_{c}\left(\mathbf{x}, t_{k}\right)\right]+n\left(\mathbf{x}, t_{k}\right)$

where $r$ is the measured voltage trace due to the echo signal and $n$ is a signal independent noise process. The shift-invariant function $h(\mathbf{x})$ is the system pulse-echo impulse response, which defines the spatial resolution, and represents acoustic transmission, scattering from a point source, and reception. The object function consists of two components: blood and tissue clutter acoustic impedances represented as $z_{b}$ and $z_{c}$ respectively, defined continuously over times $t$ and discretely over spatial locations $\mathbf{x}=\left[\begin{array}{llll}\mathbf{x}_{1} & \mathbf{x}_{2} & \ldots & \mathbf{x}_{\mathrm{M}}\end{array}\right]^{\mathrm{t}} \quad \Omega_{\mathrm{x}}$ of measure $X$, and where each sub-vector $\mathbf{x}_{\mathrm{k}}$ defines a spatial coordinate point. The operation of convolution with $h$ may alternatively be represented in operator notation as H. Hence (1) may be written as $\mathbf{r}=\mathrm{H} z_{b}+\mathrm{H} z_{c}+\mathbf{n}$, where $\mathbf{r}$ is an RF data vector for the experiment. The signal model is graphically illustrated in Fig. 2.

Spatial Fourier Domain. It will be convenient to study the problem in the spatial Fourier Domain: 


$$
R\left(\mathbf{u}, t_{k}\right)=H(\mathbf{u})\left[Z_{b}\left(\mathbf{u}, t_{k}\right)+Z_{c}\left(\mathbf{u}, t_{k}\right)\right]+N\left(\mathbf{u}, t_{k}\right) .
$$

One advantage of this form is that that for large image sizes, a shift-invariant system and WSS object functions, the Fourier transform is a Karhunen-Loeve transformation that uncouples signals in different spatial frequency channels $\mathbf{u}_{k}$. We will show later that the blood may be treated as a non-stationary process in special cases. $R$ will often be represented in vector form $\mathbf{R}=\left[R\left(\mathbf{u}_{1}, t_{1}\right), \ldots, R\left(\mathbf{u}_{1}, t_{L}\right), \ldots, R\left(\mathbf{u}_{M}, t_{1}\right), \ldots, R\left(\mathbf{u}_{M}, t_{L}\right)\right]^{t}$.

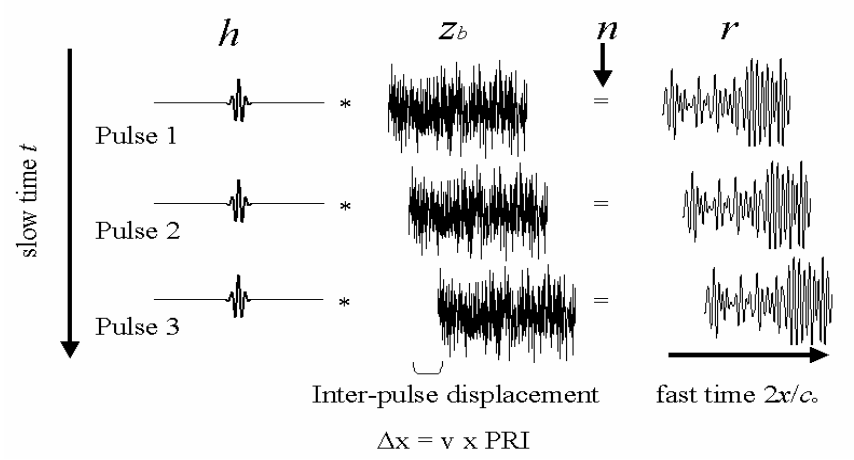

Fig. 2. Illustration of the linear systems model (1) neglecting clutter.

Assumptions. Our model makes a number of assumptions besides linear shift-invariance. We assume that spatial sampling is done sufficiently densely such that the discrete object function over space is a reasonable model. We assume that echo signals have negligible interference from echoes due to previous pulses. This is reasonable because round trip signal attenuation in tissue is typically sufficiently low compared to the signals of interest and compared to the dynamic range of the hardware. We also assume that the object moves negligibly during the signal acquisition time due to one pulse within an ensemble. Thus, we only consider translational velocities that are much less than the speed of sound $c_{0}$. The examples in this paper will use a 1-D model for simplicity. In this case the coordinate $\mathbf{x}_{\mathrm{k}}$ is simply a scalar position $x_{\mathrm{k}}$. The disadvantage of the 1-D model is that we must neglect the three-dimensional nature of the beam. Despite our focus on 1-D models, we will nevertheless keep the discussion general, and assume that $\mathbf{x}_{\mathrm{k}}$ may be a vector coordinate to allow for fast acquisition schemes and future applications. A more complete linear systems model that allows for the 3-D beam properties, shift variance, and more complex motion within an image acquisition sequence is discussed in [6]. The purpose of the simple models used in this paper is to gain intuition and analytically tractable results.

We will assume that the background clutter represented by the object function $z_{c}$ is a wide-sense stationary (WSS) stochastic process over space and over slow time, as is the noise $n$. In practice, objects heterogeneous on a scale larger than the transmitted pulse 
volume are partitioned in to locally wide-sense stationary regions. Tumor vessels are small compared with the pulse volume. We also assume that the tissue motion is WSS on the time scale of the measurement, and hence that tissue motion can be characterized by autocorrelations or power spectra. This requires that tissue motion patterns remain approximately constant over the interrogation time $L x P R I$, and that accelerations are minimal. This is a reasonable assumption even for arterial flows with a $1 \mathrm{~Hz}$ cardiac frequency when $P R F>1 \mathrm{KHz}$ and the ensemble size is $L<10$. Finally, we assume that blood, clutter, and noise processes are all statistically independent of each other.

\subsection{Cross-Spectral Density Matrix}

If $\mathrm{X}$ is the support region volume of the image, the cross-spectral density matrix $\mathbf{Q}$ is defined in a way similar to covariance matrices. Here we wish to consider the crossspectral density due to clutter and noise:

$$
\mathbf{Q}_{c+n}=X^{2}\left\langle\mathbf{R}_{c+n} \mathbf{R}_{c+n}^{\dagger}\right\rangle,
$$

where elements of $\mathbf{R}_{c+n}$ are given as $R_{c+n}\left(\mathbf{u}, t_{k}\right)=H(\mathbf{u}) Z_{c}\left(\mathbf{u}, t_{k}\right)+N\left(\mathbf{u}, t_{k}\right)$. For WSS clutter and noise processes, the cross-spectral density matrix is block diagonal:

$$
\mathbf{Q}_{c+n}=\left[\begin{array}{cccc}
\mathbf{Q}_{c+n}\left(\mathbf{u}_{1}\right) & & & 0 \\
& \mathbf{Q}_{c+n}\left(\mathbf{u}_{2}\right) & & \\
& & \ddots & \\
0 & & & \mathbf{Q}_{c+n}\left(\mathbf{u}_{M}\right)
\end{array}\right]
$$

where $\mathbf{Q}_{c+n}\left(\mathbf{u}_{k}\right)$ is an $L x L$ matrix with elements given by

$$
Q_{c+n}\left(t_{\mu}, t_{v} \mid \mathbf{u}_{k}\right)=\left|H\left(\mathbf{u}_{k}\right)\right|^{2} X^{2}\left\langle Z_{c}\left(\mathbf{u}_{k}, t_{\mu}\right) Z_{c}^{*}\left(\mathbf{u}_{k}, t_{v}\right)\right\rangle+S_{n} \delta\left(t_{\mu}-t_{v}\right) .
$$

Here we have assumed that electronic noise can be modeled as a white Gaussian noise process, and that $S_{\mathrm{n}}$ is the (flat) noise power spectrum.

\section{Detection Performance}

Equipped with the linear systems model, and the cross-spectral density structures, we now call on the tools of statistical decision theory to address the task: detection of blood that is perfusing through tissue (positive ' + ' hypothesis) from region with no blood or negligibly weak blood (null '-'hypothesis). This task could be important for distinguishing perfused 
regions of a tumor from necrotic regions. The degree of perfusion heterogeneously may be correlated with metastatic potential. It is also one step towards the more complex task of discriminating two types of flow patterns. We also assume that the background clutter and the noise are random processes but that blood can be modeled as one deterministic realization of a random process. A similar model has been used for static target discrimination in ultrasound [7]. Likelihood models for the spatial frequency domain data are normally distributed under the assumptions of [8]:

$$
\begin{aligned}
& p d f(\mathbf{R} \mid+)=X^{2 L M} \frac{\exp \left[-\frac{X^{2}}{2}\left(\mathbf{R}-\overline{\mathbf{R}}_{+}\right)^{\dagger} \mathbf{Q}_{c+n}^{-1}\left(\mathbf{R}-\overline{\mathbf{R}}_{+}\right)\right]}{\sqrt{(2 \pi)^{L M} \operatorname{det}\left(\mathbf{Q}_{c+n}\right)}} \\
& p d f(\mathbf{R} \mid-)=X^{2 L M} \frac{\exp \left[-\frac{X^{2}}{2}\left(\mathbf{R}-\overline{\mathbf{R}}_{-}\right)^{\dagger} \mathbf{Q}_{c+n}^{-1}\left(\mathbf{R}-\overline{\mathbf{R}}_{-}\right)\right]}{\sqrt{(2 \pi)^{L M} \operatorname{det}\left(\mathbf{Q}_{c+n}\right)}}
\end{aligned}
$$

Here the mean values for our task are:

$\bar{R}_{+}\left(\mathbf{u}_{k}, t_{l}\right)=\left\langle R_{+}\left(\mathbf{u}_{k}, t_{l}\right)\right\rangle_{n}=B\left(\mathbf{u}_{k}, t_{l}\right) \equiv H\left(\mathbf{u}_{k}\right) Z_{b}\left(\mathbf{u}_{k}, t_{l}\right)$ and $\bar{R}_{-}\left(\mathbf{u}_{k}, t_{l}\right)=0$.

The ideal observer test statistic obtained from the log-likelihood ratio is thus:

$$
\lambda=\Delta \overline{\mathbf{R}}^{\dagger} \mathbf{Q}_{c+n}^{-1} \mathbf{R}
$$

where $\Delta \overline{\mathbf{R}}=\overline{\mathbf{R}}_{+}-\overline{\mathbf{R}}_{-}=\mathbf{B}$. This test statistic is linear in the data and for the situation at hand is equivalent to a Hotelling observer [9]. The strategy is to perform matched filtering of the time-evolving signal with a pre-whitening step. Pre-whitening over the slow time spectral domain means weighting the Doppler Fourier amplitudes $\mathbf{R}$ by the factor $\Delta \overline{\mathbf{R}}^{\dagger} \mathbf{Q}_{c+n}^{-1}$ that maximizes coefficients least influenced by clutter and noise. Performance is predicted by

$$
S N R_{I}^{2}=\Delta \overline{\mathbf{R}}^{\dagger} \mathbf{Q}_{c+n}^{-1} \Delta \overline{\mathbf{R}}=\mathbf{B}^{\dagger} \mathbf{Q}_{c+n}^{-1} \mathbf{B} .
$$

Using the approach of [7], we average over random realizations of the blood to obtain

$$
\left\langle S N R_{I}^{2}\right\rangle=\operatorname{tr}\left\langle\left\langle\mathbf{B B}^{\dagger}\right\rangle \mathbf{Q}_{c+n}^{-1}\right\}=\operatorname{tr}\left\{\mathbf{Q}_{b} \mathbf{Q}_{c+n}^{-1}\right\},
$$

where $\mathbf{Q}_{b}=\left\langle\mathbf{B B} \mathbf{B}^{\dagger}\right\rangle$. When $\mathbf{Q}_{b}$ and $\mathbf{Q}_{c+n}^{-1}$ are simultaneously diagonalizable, by the same Karhunen-Loeve eigendecomposition, the $S N R$ expression reduces to 
$\left\langle S N R_{I}^{2}\right\rangle=\operatorname{tr}\left\{\boldsymbol{\Lambda}_{b}\left[\boldsymbol{\Lambda}_{c}+\boldsymbol{\Lambda}_{n}\right]^{-1}\right\}$, where the $\Lambda^{\prime}$ s are diagonal eigenvalue matrices corresponding to blood, clutter and noise cross-spectral density matrices respectively.

\subsection{Large Ensemble Size}

When $L$ is large, and adequate slow time resolution exists, the Karhunen-Loeve transformation reduces to a temporal DFT. In this situation aliasing is largely avoided. The $S N R$ can then be written as

$$
S N R_{G N E Q}^{2} \equiv\left\langle S N R_{I}^{2}\right\rangle_{b}=\sum_{k=1}^{N} \int d \mathbf{u} \frac{\left\langle\left|\Delta Z_{b}\left(\mathbf{u}, f_{k}\right)\right|^{2}\right\rangle_{b}|H(\mathbf{u})|^{2}}{|H(\mathbf{u})|^{2} S_{c}\left(\mathbf{u}, f_{k}\right)+S_{n}\left(\mathbf{u}, f_{k}\right)} .
$$

This is a generalization of the Wagner-Brown theory of detectability [10] applied to Doppler ultrasound systems. $S_{c}$ and $S_{n}$ are the clutter and noise power spectra respectively. The power spectra are in the spatial frequency $(\mathbf{u})$, temporal-frequency $(f)$ domain. The object contrast or "task" is defined as

$$
S_{\Delta B}\left(\mathbf{u}, f_{k}\right)=\left\langle\left|\Delta Z_{b}\left(\mathbf{u}, f_{k}\right)\right|^{2}\right\rangle_{b} / X
$$

and represents the power spectrum of the difference between blood object functions of hypothesis (+) and hypothesis (-) signals. This is not the same thing as the difference of power spectral densities. The $S N R_{G N E Q}$ is dependent on the differential blood object power spectrum to make a decision about flow being present or absent (normal or abnormal). The quantity that weights the blood object power spectrum in the integration is like a generalized version of Noise Equivalent Quanta $(N E Q)$, a measure commonly used to characterize system performance in photon imaging modalities. In our situation, the generalized noise equivalent quanta $(G N E Q)$ is defined as

$$
\operatorname{GNEQ}\left(\mathbf{u}, f_{k}\right)=\frac{|H(\mathbf{u})|^{2}}{|H(\mathbf{u})|^{2} S_{c}\left(\mathbf{u}, f_{k}\right)+S_{n}\left(\mathbf{u}, f_{k}\right)}
$$

and characterizes the system, noise and clutter in a blood flow independent way. $N E Q$ for photon imaging systems represents the fraction of photons that contribute information. For ultrasound, GNEQ represents the fraction of speckle energy that carries information. In photon imaging systems, the ideal observer detection signal to noise ration is an integration of $N E Q$ weighted by the Fourier magnitude of the target signal. Acoustic physics dictates that the mean of the object is not the important feature for detection using ultrasound, rather the variance or spatial fluctuations in the object function. Importantly, GNEQ can be measured for a particular system, tissue type, and tissue motion pattern 
since all of its components are measurable quantities. Curiously, $H$ in this framework only depends on spatial frequencies, and not temporal frequencies: this is because all motion dependence is due to the object and not to the system.

\subsection{Limited Ensemble Size}

In general, simultaneous diagonalization of $\mathbf{Q}_{b}$ and $\mathbf{Q}_{c+n}^{-1}$ is not possible. For small ensemble sizes, and wide-sense stationary statistics, the cross-spectral density matrices are Toeplitz, but not well approximated by block-circulant matrices, and hence are not diagonalizable by a DFT. Since most Power and Color ultrasound systems use only 4-20 pulses, consideration of the limited ensemble size scenario is of great practical importance. We consider here specific analytically tractable models that may nevertheless be of considerable interest for tumor flow and perfusion imaging.

Clutter Model. Tissue motion is often minimal for tumor imaging. If we assume the clutter does not move, and remains correlated over the entire pulse train interrogation, then it can be shown that the clutter cross-spectral density has the block diagonal form of (4), with block-diagonal elements given as

$$
Q_{c+n}\left(t_{\mu}, t_{v} \mid \mathbf{u}_{k}\right)=\left|H\left(\mathbf{u}_{k}\right)\right|^{2} S_{c}\left(\mathbf{u}_{k}\right)+S_{n} \delta\left(t_{\mu}-t_{v}\right) .
$$

$\mathbf{Q}_{c+n}\left(\mathbf{u}_{k}\right)$ is the sum of uniform matrix (a matrix with constant values everywhere) and a diagonal matrix. It has an analytically tractable inverse

$$
\mathbf{Q}_{c+n}^{-1}\left(\mathbf{u}_{k}\right)=\frac{1}{L\left|H\left(\mathbf{u}_{k}\right)\right|^{2} S_{c}\left(\mathbf{u}_{k}\right)+S_{n}}\left[\begin{array}{cccc}
p & q & \cdots & q \\
q & p & & \\
\vdots & & \ddots & \\
q & & & p
\end{array}\right],
$$

where $p=\left|H\left(\mathbf{u}_{k}\right)\right|^{2} \frac{S_{c}\left(\mathbf{u}_{k}\right)}{S_{n}}[L-1]+1$ and $q=-\left|H\left(\mathbf{u}_{k}\right)\right|^{2} \frac{S_{c}\left(\mathbf{u}_{k}\right)}{S_{n}}$. Block diagonal matrices are small (only $L x L$ ) so computational approaches to inversion would also be an attractive future direction.

Blood Model. As a first step to understanding more complicated flow models, we consider an object model for blood that is a random process moving with translational velocity $\mathbf{v}_{\mathrm{b}}$ : 


$$
z_{b}(\mathbf{x}, t)=z_{b}\left(\mathbf{x}-\mathbf{v}_{b} t\right) .
$$

In the spatial-frequency domain, this can be written as:

$$
Z_{b}(\mathbf{u}, t)=e^{-i 2 \pi t \mathbf{u} \cdot \mathbf{v}_{b}} Z_{b}(\mathbf{u})
$$

If blood is a non-stationary process then the cross-spectral density matrix may not be of block-diagonal form because of spatial frequency correlations. However, only the block diagonal elements of the cross-spectral density matrix are important for the trace (10). The block diagonal elements are given as:

$$
Q_{b}\left(t_{\mu}, t_{v} \mid \mathbf{u}_{k}\right)=X^{2}\left\langle B\left(\mathbf{u}_{k}, t_{\mu}\right) B^{*}\left(\mathbf{u}_{k}, t_{\eta}\right)\right\rangle=\left|H\left(\mathbf{u}_{k}\right)\right|^{2} S_{Z_{b}}\left(\mathbf{u}_{k}\right) e^{-i 2 \pi\left[t_{\mu}-t_{\eta}\right] \mathbf{u}_{k} \cdot \mathbf{v}_{b}} .
$$

If the blood object function can be modeled as a wide-sense stationary white gaussian noise process $\sim N\left(0, \sigma^{2} \mathbf{I}\right)$, windowed by a window $w(\mathbf{x})$, it can be shown that

$$
S_{Z_{b}}\left(\mathbf{u}_{k}\right)=\sigma^{2} \int d \mathbf{x}|w(\mathbf{x})|^{2}=\sigma^{2} A
$$

where $A$ is the area of the perfused region.

Another important aspect of the blood model is decorrelation over time. Microvascular networks can have tortuous and multidirectional flow patterns within an ultrasonic sample volume that makes velocity estimation difficult. One way of modeling this complex situation is to guess that the collection of blood scatterers decorrelates over time, and that the decorrelation rate is proportional to the flow rate, and hence metabolic demand. One model for temporal decorrelation is a complex exponential decay. As a phenomenological addendum to (18), we hence postulate the cross-spectral density model

$$
Q_{b}\left(t_{\mu}, t_{v} \mid \mathbf{u}_{k}\right)=\left|H\left(\mathbf{u}_{k}\right)\right|^{2} S_{Z_{b}}\left(\mathbf{u}_{k}\right) e^{-i 2 \pi\left[t_{\mu}-t_{\eta}\right] \mathbf{u}_{k} \cdot \mathbf{v}_{b}} e^{-\alpha\left|t_{\mu}-t_{\eta}\right|}
$$

where $\alpha$ is the decorrelation parameter. Gaussian decorrelation models and other diffusion-based models are also possible. Signal decorrelation may be due to object correlation or due scattering regions moving out of the ultrasonic sample volume. In the latter case the decorrelation parameter may depend on the velocity beam properties.

SNR. Evaluating the trace (10), the ideal observer performance metric for stationary clutter and moving, decorrelating blood can be shown to be

$$
S N R_{I}^{2}=\int d \mathbf{u} \frac{|H(\mathbf{u})|^{2} S_{Z_{b}}(\mathbf{u})}{L|H(\mathbf{u})|^{2} S_{c}(\mathbf{u})+S_{n}}\left[\frac{S_{c}(\mathbf{u})}{S_{n}}|H(\mathbf{u})|^{2} \Gamma(\mathbf{u})+L\right],
$$


where

$$
\Gamma(\mathbf{u})=L(L-1)-2 \operatorname{Re}\left\{\sum_{m=1}^{L}(L-m) \alpha^{m}\right\},
$$

and $\alpha=e^{-\alpha|\Delta t|} e^{-i 2 \pi \Delta t \mathbf{u} \cdot \mathbf{v}_{b}}$. Here $\Delta t$ is the pulse repetition interval (PRI). The series can be evaluated as the sum of a geometric series and the derivative of a geometric series:

$$
\sum_{m=1}^{L}(L-m) \alpha^{m}=L\left[\frac{\alpha-\alpha^{L+1}}{1-\alpha}\right]-\alpha\left[\frac{L \alpha^{L+1}-(L+1) \alpha^{L}+1}{(1-\alpha)^{2}}\right] .
$$

When there is no decorrelation, and zero blood velocity, $\Gamma(\mathbf{u})$ vanishes, leaving

$$
S N R_{I}^{2}=\int d \mathbf{u} \frac{|H(\mathbf{u})|^{2} S_{Z_{b}}(\mathbf{u})}{|H(\mathbf{u})|^{2} S_{c}(\mathbf{u})+S_{n} / L},
$$

which is similar to expressions obtained previously, except that the noise power is lowered by a factor of $L$. This makes sense: the task is now to simply detect additive, unmoving blood from a random background, and there are $L$ images averaged. Hence, the noise power should decrease as there are more images averaged. For $L=1$, the expression corresponds precisely to the lesion detection SNR of Ref. [7], Eq. 23.

Clearly then, all the information about motion is contained in the function $\Gamma$, which depends on the PRI, decorrelation parameter, velocity, ensemble size, and spatial frequency vector. Aside from the $P R I$ and the ensemble size, $\Gamma$ is independent of the system, and represents the information about blood flow and perfusion. The $\mathrm{SNR}^{2}$ of (21) can thus be interpreted as the sum of a time-dependent term and a time-independent term. The time-dependent term gives all the information about motion and decorrelation. The time-independent term describes static detection.

\section{Results}

To gain more intuition regarding the role of motion, we computationally evaluated (21) with various system and motion parameters for a 1-D system where the system $p s f$ is modeled as a Gaussian weighted sinusoid. We modeled coherent motion as being along the direction of pulse propagation. Results are illustrated in Fig. 3. The system bandwidth was varied while maintaining the total pulse energy constant. Fig. 3(a) shows that bandwidth is relatively unimportant as long as the same amount of total energy is employed. Fig. 3(b) shows that detectability is lowest when there is no motion. The ideal observer uses motion information to help the detection process. There are repeating nulls 
every $250 \mathrm{~mm} / \mathrm{s}$. This is the point where $P R I \times 2 f_{\mathrm{o}} / c \times v_{\mathrm{b}}=1$. Note that $2 f_{\mathrm{o}} / c$ is the spatial frequency at which there is greatest system sensitivity. As velocity is increased, blood power shifts away from the clutter power until it is aliased back into the clutter spectrum, giving the null at $250 \mathrm{~mm} / \mathrm{s}$ etc. As expected, the detectability improves with $L$, and Fig. 3(c) demonstrates that this relationship is nearly linear. As the PRI is increased, $S N R$ is also improved (results not shown). Of course transient flow conditions may prevent one from using long PRI's or large ensemble sizes if temporal resolution is desired. One interesting point is that decorrelation of blood is actually helpful to signal detection (Fig. 3(d).). The ideal observer is most interested with how the signal changes over time, hence the more change in the observation period the better. Thus decorrelation and velocity shift spectral energy of blood away from the DC region where clutter obscures detection. Velocity shifts can however be aliased back over the clutter region once again hampering the task. Large velocities are necessary to induce substantial aliasing, and these speeds are not physiological in tumors. Hence if decorrelation is well sampled, the large ensemble limit of section 3.2 may be a reasonable framework for studying tumor perfusion.

(a)

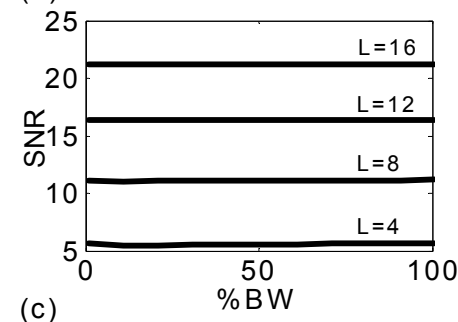

$$
\text { (c) }
$$

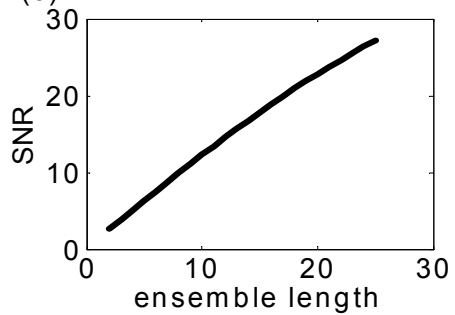

(b)

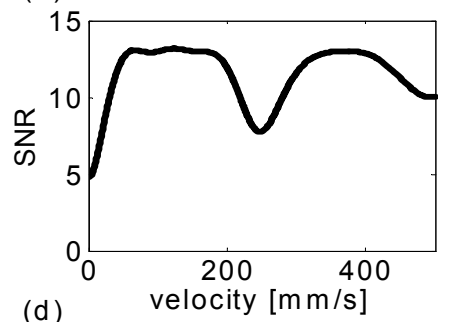

(d)

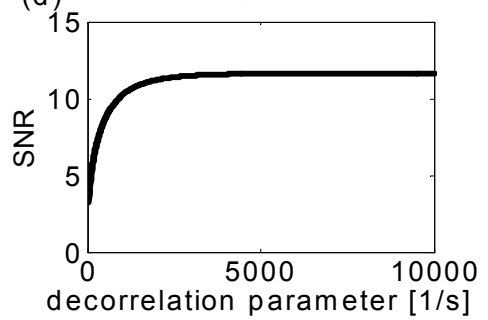

Fig. 3. Detection performance SNR as a function of (a) bandwidth, maintaining total pulse energy constant (b) velocity (c) ensemble length and (d) decorrelation parameter. Parameters were, $f_{\mathrm{o}}=3$ $\mathrm{MHz}, B W=80 \%, c=1500 \mathrm{~m} / \mathrm{s}, L=8$ pulses, $P R F=1 \mathrm{KHz} ., v=5 \mathrm{~mm} / \mathrm{s}$, and $\alpha=1 / P R I$ unless otherwise specified. The clutter to noise and blood to noise ratios were 38.8 and 1.94 respectively.

\section{Conclusions}

An ideal observer model for detecting perfusing blood in a tissue background has been developed. When decorrelation is adequately sampled (large enough ensemble size), and 
when velocities are low, the ideal observer signal to noise ratio depends on a quantity similar to Noise Equivalent Quanta (NEQ) used in photon imaging modalities. Coherent motion and temporal decorrelation of blood were modeled in a simple 1-D ultrasound system. The system detection performance for a simple model was shown to be the sum of motion-dependent and motion-independent terms. This model included the effects of aliasing. Decorrelation and translational velocity of blood were shown to be advantageous to the task. The ideal observer model, assumed that velocities and decorrelation rates were known a priori. Task performance may change when the velocity is unknown. The theoretical framework here is a starting point for future work in the area of performance assessment of Power Doppler systems.

\section{References}

${ }^{1}$ J. A. Jensen, Estimation of Blood Velocities Using Ultrasound: A Signal Processing Approach. New York: Cambridge Univ. Press, 1996.

${ }^{2}$ K. W. Ferrara, C. W. Merritt, P. N. Burns, F. S. Foster, R. F. Mattrey, S. A. Wickline, "Evaluation of Tumor Angiogenesis with US: Imaging, Doppler, and Contrast Agents," Acad. Radiol. Vol. 7, pp. 824-839, 2000.

${ }^{3}$ C. Kasai, K. Namekawa, A. Koyano, R. Omoto, "Real-time two-dimensional blood flow imaging using an autocorrelation technique. IEEE Trans. Sonics Ultrason. SU-32, pp. 458-464.

${ }^{4}$ G. E. Trahey, J. W. Allison, and O. T. von Ramm, "Angle independent ultrasonic detection of blood flow," IEEE Trans. Biomed. Eng. Vol. 34, pp. 965-967, 1987.

${ }^{5}$ J. M. Rubin, R. O. Bude, P. L. Carson, R. L. Bree, R. S. Alder, "Power Doppler US: A Potentially Useful Alternative to Mean-Frequency-based Color Doppler US," Radiology, Vol. 190, pp. 853-856, 1994.

${ }^{6}$ R. J. Zemp, C. K. Abbey, M. F. Insana, "Linear system models of ultrasound imaging: Application to signal statistics," IEEE Trans. Ultrason., Ferroelect., Freq. Contr., To be published.

${ }^{7}$ R. J. Zemp, C. K. Abbey, M. F. Insana, "Generalized NEQ for assessment of ultrasound image quality," Proc. SPIE Medical Imaging, San Diego, CA, 2003.

${ }^{8}$ R. F. Wagner, M. F. Insana, and D. G. Brown, "Statistical properties of radio-frequency and envelope-detected signals with applications to medical ultrasound," J. Opt. Soc. Am. A, Vol. 4, pp. 910-922, 1987.

${ }^{9}$ H. H. Barrett, "Objective assessment of image quality: effects of quantum noise and object variability," J. Opt. Soc. Am. A, Vol. 7, pp. 1266-1278, 1990.

${ }^{10}$ R. F. Wagner and D. G. Brown, "Unified SNR analysis of medical imaging systems," Phys. Med. Biol. Vol. 30, pp. 489-518, 1985. 